\title{
Autophagy induction in the skeletal myogenic differentiation of human tonsil-derived mesenchymal stem cells
}

\author{
SAEYOUNG PARK ${ }^{1 *}$, YOONYOUNG CHOI $^{1 *}$, NAMHEE JUNG $^{1}$, JIEUN KIM $^{1}$, SEIYOON OH $^{2}$, \\ YEONSIL YU $^{3}$, JUNG-HYUCK AHN ${ }^{1}$, INHO JO ${ }^{3}$, BYUNG-OK CHOI ${ }^{4}$ and SUNG-CHUL JUNG ${ }^{1}$ \\ ${ }^{1}$ Department of Biochemistry, School of Medicine, Ewha Womans University, Seoul 07985, Republic of Korea; \\ ${ }^{2}$ Department of Human Biology, College of Human Ecology, Cornell University, Ithaca, NY 14850, USA; \\ ${ }^{3}$ Department of Molecular Medicine, School of Medicine, Ewha Womans University, Seoul 07985; \\ ${ }^{4}$ Department of Neurology, Samsung Medical Center, Sungkyunkwan University, Seoul 06351, Republic of Korea
}

Received July 29, 2016; Accepted February 14, 2017

DOI: $10.3892 / \mathrm{ijmm} .2017 .2898$

\begin{abstract}
Mesenchymal stem cells (MSCs) are capable of selfrenewal and differentiation and are thus a valuable source for the replacement of diseased or damaged organs. Previously, we reported that the tonsils can be an excellent reservoir of MSCs for the regeneration of skeletal muscle (SKM) damage. However, the mechanisms involved in the differentiation from tonsil-derived MSCs (T-MSCs) to myocytes via myoblasts remain unclear. To clarify these mechanisms, we analyzed gene expression profiles of T-MSCs during differentiation into myocytes compared with human skeletal muscle cells (hSKMCs). Total RNA was extracted from T-MSCs, T-MSCderived myoblasts and myocytes, and hSKMCs and was subjected to analysis using a microarray. Microarray analysis of the three phases of myogenic differentiation identified candidate genes associated with myogenic differentiation. The expression pattern of undifferentiated T-MSCs was distinguishable from the myogenic differentiated T-MSCs and hSKMCs. In particular, we selected FNBP1L, which among the upregulated genes is essential for antibacterial autophagy, since autophagy is related to SKM metabolism and myogenesis. T-MSCs differentiated toward myoblasts and skeletal myocytes sequentially, as evidenced by increased expression of autophagy-related markers (including Beclin-1, LC3B and Atg5) and decreased expression of Bcl-2. Furthermore, we reconfirmed that autophagy has an effect on the mechanism of skeletal myogenic differentiation derived from T-MSCs by treatment with 5 -azacytidine and bafilomycin A1. These data suggest that the transcriptome of the T-MSC-derived
\end{abstract}

Correspondence to: Dr Sung-Chul Jung, Department of Biochemistry, School of Medicine, Ewha Womans University, 1071 Anyangcheon-ro, Yangcheon-gu, Seoul 07985, Republic of Korea

E-mail: jungsc@ewha.ac.kr

*Contributed equally

Key words: human tonsil-derived mesenchymal stem cells, skeletal myocyte, transcriptome, differentiation, autophagy myocytes is similar to that of hSKMCs, and that autophagy has an important role in the mechanism of myogenic differentiation of T-MSCs.

\section{Introduction}

Mesenchymal stem cells (MSCs) are capable of self-renewal and differentiation, and are therefore a valuable source of cells for the replacement of diseased or damaged organs. Compared with embryonic stem cells or induced pluripotent stem cells, MSCs have an ability to regulate the immune system and do not form teratomas. They can be isolated from various sources, including the tonsils $(1,2)$, bone marrow (3), adipose tissue (4), amniotic fluid (5), umbilical cord blood (6), the placenta (7) and dental pulp (8). Previously, we reported that tonsil-derived MSCs (T-MSCs) can be differentiated into skeletal myocytes, and are therefore a promising candidate in cell therapies for skeletal muscle (SKM)-related disease (2). However, the mechanisms of differentiation from T-MSCs to myocytes remain unclear.

Myogenic differentiation is regulated by a variety of biochemical pathways, including those involving growth factors fibroblast growth factor (FGF), transforming growth factor- $\beta$ (TGF- $\beta$ ), insulin-like growth factor 1 (IGF1) (9), Janus kinase 2/signal transducer and activator of transcription (STAT)2/STAT3 (10), Notch (11), ER stress, and autophagy (12). FGF-2 and HGF promote proliferation of myogenic progenitors and delay their differentiation, in part by inhibiting the expression of myogenic regulatory factors, such as MyoD $(13,14)$. IGF1 promotes myogenic differentiation and enhances protein synthesis in differentiated myofibers by activating the translation factor $4 \mathrm{E}-\mathrm{BP}$ and the ribosomal protein S6 kinase (p70S5K), and by inhibiting muscle-specific E3 ligases that promote protein degradation (15). ER stress has a positive effect on myofiber formation in vitro, possibly mimicking the action of signals that drive apoptosis and differentiation in vivo (16). Recently, it was shown that autophagy is induced during muscle differentiation despite the concomitant activation of mammalian target of rapamycin (mTOR) using the mouse myoblast cell line, $\mathrm{C} 2 \mathrm{C} 12$ (12). Autophagy plays an essential role in cellular development and differentiation (17), 
and human bone marrow MSCs may differentiate to become early osteocytes and adipocytes by consumption of autophagosomes (18). In addition, mitophagy, the selective degradation of mitochondria by autophagy, has been reported to be required for mitochondrial biogenesis and myogenic differentiation of C2C12 myoblasts (19).

In the present study, we analyzed transcriptomes using microarrays and then performedlarge-scale expression profiling of T-MSCs during differentiation into myocytes compared with hSKMCs and the candidate genes identified as associated with myogenic differentiation. Immunocytochemistry, reverse transcriptase-polymerase chain reaction (RT-qPCR), and western blotting confirmed the pathway of interest that was obtained from the candidate gene. The results suggest that autophagy has an important role in the myogenic differentiation of the T-MSCs.

\section{Materials and methods}

Ethics statement. The Institutional Review Board(ECT-11-53-02) of Ewha Womans University Mokdong Hospital (Seoul, Korea) approved all of the experimental procedures used in this study. Informed written consent was secured from each patient and/or their legal representatives.

Isolation of T-MSCs. Isolation of T-MSCs from tonsil tissue was performed as described previously $(1,2)$. Tonsillar tissues were extracted during tonsillectomy, and minced and digested in Dulbecco's modified Eagle's medium (DMEM) containing $210 \mathrm{U} / \mathrm{ml}$ collagenase type I (both from Invitrogen, Carlsbad, CA, USA) and DNase (10 mg/ml; Sigma-Aldrich, St. Louis, MO, USA). After cells were passed through a cell strainer (BD Biosciences, San Jose, CA, USA), mononuclear cells were obtained by Ficoll-Paque (GE Healthcare, Chalfont St. Giles, UK) density gradient centrifugation. Cells were cultured for $48 \mathrm{~h}$ at $37^{\circ} \mathrm{C}$ in low-glucose DMEM containing $10 \%$ fetal bovine serum (FBS; Invitrogen) and $1 \%$ penicillin/streptomycin (Sigma-Aldrich) in a humidified chamber with $5 \% \mathrm{CO}_{2}$, followed by removal of nonadherent cells and a supply of fresh medium for the T-MSCs. These freshly cultured cells were expanded over 3-5 passages and subsequently used in the present study.

Differentiation. To induce the myogenic differentiation of the T-MSCs, $3-4 \times 10^{6}$ cells were plated in a $10-\mathrm{cm}$ Petri dish in low-glucose DMEM supplemented with $10 \%$ FBS. At 1-3 days, the cells spontaneously aggregated to form spheres $50-100 \mathrm{~mm}$ in diameter. Once the spheres had formed, the medium was replaced with DMEM/nutrient mixture F-12 (DMEM/F-12; Invitrogen) supplemented with $1 \mathrm{ng} / \mathrm{ml}$ TGF- $\beta$ (R\&D Systems, Minneapolis, MN, USA), nonessential amino acids (NEAA; Invitrogen) and insulin-transferrinselenium (ITS; Gibco Life Technologies, Grand Island, NY, USA) for a further 4 days to allow their differentiation into myoblasts. The T-MSCs expanded beyond the spheres when transferred to a collagen-coated dish in the abovementioned myoblast differentiation medium, and formed a rosette-like spread. To induce terminal differentiation into myocytes, the myoblasts were cultured for 2 weeks in myogenic induction medium, which consisted of low-glucose DMEM containing $10 \mathrm{ng} / \mathrm{ml}$ IGF1 (R\&D Systems) and 2\% FBS (Fig. 1A-E).
The hSKMCs (Fig. 1F) were differentiated in serum-free medium (DMEM F-12; Invitrogen) containing 10 ng/ml IGF1 (R\&D Systems).

Chemical reagents. Autophagy was confirmed with 5-azacytidine as an inducer and bafilomycin A1 (Baf1) as an inhibitor to identify the mechanism of differentiation to myogenic cells. To produce a stock solution (1,000X), $1 \mathrm{mM} 5$-azacytidine and $1 \mathrm{mM}$ Baf1 (Sigma-Aldrich) were dissolved in $1 \mathrm{ml}$ dimethylsulfoxide in water and acetic acid, respectively. After the inducer or inhibitor was added to the differentiation medium for $1 \mathrm{~h}$, the medium was replaced with chemical reagent-free medium.

Total RNA preparation. The cells were seeded in $100-\mathrm{mm}$ culture dishes under individual culture medium. Total RNA was extracted from three replicates per group using a Qiagen (Germantown, MD, USA) RNeasy Mini kit. RNA purity and integrity were evaluated using a ND-1000 spectrophotometer (NanoDrop, Wilmington, NC, USA), and Agilent 2100 Bioanalyzer (Agilent Technologies, Palo Alto, CA, USA).

Microarray analysis. Total RNA was amplified and purified using a TargetAmp-Nano Labeling kit for Illumina Expression BeadChip (Epicentre, Madison, WI, USA) to yield biotinylated cRNA according to the manufacturer's instructions. Briefly, $200 \mathrm{ng}$ of total RNA was reverse-transcribed to cDNA using a T7-oligo(dT) primer. Second-strand cDNA was synthesized, transcribed in vitro, and labeled with biotin-NTP. After purification, the cRNA was quantified using an ND-1000 spectrophotometer. An amount $750 \mathrm{ng}$ of the labeled cRNA samples was hybridized to each Human HT-12 version 4.0 Expression BeadChip for $18 \mathrm{~h}$ at $58^{\circ} \mathrm{C}$, according to the manufacturer's instructions (Illumina Inc., San Diego, CA, USA). Detection of an array signal was achieved using Amersham fluorolink streptavidin-Cy3 (GE Healthcare Bio-Sciences) following the bead array manual. Arrays were scanned with an Illumina bead array reader confocal scanner according to the manufacturer's instructions. The quality of hybridization and overall chip performance were monitored by visual inspection of both internal quality control checks and the raw scanned data. Raw data were extracted using the software provided by the manufacturer [Illumina GenomeStudio, version 2011.1 (Gene Expression Module, version 1.9.0)]. Array probes were transformed using a logarithm and normalized using a quantile method. Statistical significance of the expression data was determined using an LPE test and fold-change in which the null hypothesis was that no difference exists between groups. False discovery rate (FDR) was controlled by adjusting the P-value using a Benjamini-Hochberg algorithm. For a DEG set, hierarchical cluster analysis was performed using complete linkage and Euclidean distance as a measure of similarity. Gene-Enrichment and Functional Annotation analysis for the significant probe list was performed using DAVID (http:// david.abcc.ncifcrf.gov/home.jsp). All data analysis and visualization of differentially expressed genes were conducted using $\mathrm{R}$ version 3.0.2 (www.r-project.org).

Real-time quantitative PCR. Total RNA was extracted from cells using a Qiagen RNeasy Mini kit. Complementary DNA 
(cDNA) was synthesized using Superscript II (Invitrogen) and oligo $(\mathrm{dT})_{20}$ primers at $42^{\circ} \mathrm{C}$ for $1 \mathrm{~h}$ followed by incubation at $72^{\circ} \mathrm{C}$ for $15 \mathrm{~min}$. From the results of the microarray analysis, we determined genes that were either upregulated or downregulated, or related to autophagy. RT-qPCR was performed using SYBR Premix Ex Taq (Takara Bio Inc., Shiga, Japan) on an ABI 7500 Fast Real-Time PCR system (PE Applied Biosystems, Foster City, CA, USA) to confirm the relative levels of expression of genes in the T-MSCs, T-MSC-derived myoblasts and myocytes. The total volume of the PCR reaction was $20 \mathrm{ml}$, containing $0.8 \mathrm{ml}$ of each primer $(5 \mathrm{mM}), 1 \mathrm{ml} \mathrm{cDNA}, 10 \mathrm{ml}$ 2X SYBR Premix Ex Taq II, $0.4 \mathrm{ml}$ Rox dye, and $7.8 \mathrm{ml}$ sterile $\mathrm{ddH}_{2} \mathrm{O}$. PCR cycling conditions were as follows: initial $30 \mathrm{sec}$ denaturation at $95^{\circ} \mathrm{C}$, followed by 40 cycles of amplification at $95^{\circ} \mathrm{C}$ for $3 \mathrm{sec}$ and $60^{\circ} \mathrm{C}$ for $30 \mathrm{sec}$, and a subsequent melting curve analysis, where the temperature was increased from 60 to $95^{\circ} \mathrm{C}$. To quantify the expression of each candidate gene, the mRNA expression levels were normalized to the level of glyceraldehyde 3-phosphate dehydrogenase (GAPDH) mRNA. Relative gene expression was analyzed using a comparative cycle threshold $(\mathrm{Ct})$ method $\left(2^{-\Delta \Delta \mathrm{Ct}}\right)(20)$. RT-qPCR was performed in triplicate for each sample and was repeated three times for each assay. Primers of the forward and reverse primers used were as follows: supervillin (SVIL) forward, 5'-GAAG TGCTCCCTTCTGCAAC-3' and reverse, 5'-AGTGCTTTGC CAGCTGAAAT-3'; sortilin 1 (SORT1) forward, 5'-TAACAG CTGCGTGGAAAGTG-3' and reverse, 5'-GCACTCCAG CCCTAACCATA-3'; angiogenin (ANG) forward, 5'-CTCACC CTGCAAAGACATCA-3' and reverse, 5'-TCCATGTAGCT TGCAAGTGG-3'; formin-binding protein (FNBP1L) forward, 5'-AAAGGTGACGGATGGACAAG-3' and reverse, 5'-TTG CCCATTTCCTCAGAAAC-3'; beclin-1 forward, 5'-AGG TTGAGAAAGGCGAGACA-3' and reverse, 5'-GCTTTTGT CCACTGCTCCTC-3'; autophagy-related 5 (Atg5) forward, 5'-AAAGATGTGCTTCGAGATGTGT-3' and reverse, 5'-CAC TTTGTCAGTTACCAACGTCA-3'; autophagy-related 3 (Atg3) forward, 5'-GACCCCGGTCCTCAAGGAA-3' and reverse, 5'-TGTAGCCCATTGCCATGTTGG-3'; autophagy-related 14 (Atg14) forward, 5'-GCGCCAAATGCGTTCAGAG-3' and reverse, 5'-AGTCGGCTTAACCTTTCCTTCT-3'; GAPDH forward, 5'-ACACCCACTCCTCCACCTTT-3' and reverse, 5'-TGCTGTAGCCAAATTCGTTG-3'.

Immunocytochemistry. The cells grown on coverslips were fixed in $4 \%(\mathrm{v} / \mathrm{v})$ paraformaldehyde (Sigma-Aldrich) for $15 \mathrm{~min}$ at room temperature or overnight at $4^{\circ} \mathrm{C}$. After rinsing in PBS, the fixed cells were permeabilized and nonspecific epitopes were blocked using $2 \%$ bovine serum albumin (Bovogen Biologicals, Keilor East, Vic, Australia) in $0.1 \%$ Tween-20/PBS, followed by incubation in the diluted primary antibody for $1 \mathrm{~h}$ at room temperature or overnight at $4^{\circ} \mathrm{C}$. After three washes in PBS, samples were incubated for $1 \mathrm{~h}$ at room temperature with secondary antibodies diluted in PBS. Prepared samples were then mounted using Vectashield medium containing 4',6-diamidino-2-phenylindole (DAPI; Vector Laboratories, Burlingame, CA, USA) and photographed using a fluorescence microscope (Nikon Corporation, Tokyo, Japan). The manufacturers and catalog numbers of the antibodies employed were as follows: mouse anti-myosin heavy chain (MHC; cat. no. MAB4470; R\&D Systems) mouse anti- myogenin (cat. no. ab-1835; Abcam, Cambridge, MA, USA), mouse anti-desmin (cat. no. D1033; Sigma-Aldrich, St. Louis, MO, USA), Alexa-568 goat anti-mouse IgG (cat. no. A-11004), Alexa-488 goat anti-rabbit IgG (cat. no. A-11008) (both from Life Technologies). Quantification of immunofluorescence staining confirmed that four slides were used for each condition. Graphs represent the average of multiple tests from three independent experiments.

Western blotting. Protein concentration was determined using the Bradford assay reagent (Bio-Rad, Hercules, CA, USA) after lysing cells in Pro-Prep buffer (Intron Biotechnology, Seongnam, Korea) supplemented with phosphatase inhibitor cocktail solution (Dawinbio, Hanam, Korea). The cells were washed with ice-cold PBS and exposed to Pro-Prep buffer supplemented with phosphatase inhibitor cocktail solution for $30 \mathrm{~min}$ on ice. Insoluble material was removed by centrifugation at $12,000 \mathrm{x} \mathrm{g}$ for $10 \mathrm{~min}$ at $4^{\circ} \mathrm{C}$. Proteins (30-80 mg) were separated by $7.5-13.5 \%$ sodium dodecyl sulfate-polyacrylamide gel electrophoresis and transferred to a polyvinylidene fluoride or nitrocellulose membrane (Millipore, Billerica, MA, USA). The membranes were blocked with 5\% skim milk in Tris-buffered saline containing $0.1 \%$ Tween 20 (TBST) for $2 \mathrm{~h}$ at room temperature. The blots were then incubated with primary antibody overnight at $4^{\circ} \mathrm{C}$. Antibodies used for western blot analysis were rabbit anti-LC3B (cat. no. 14600-1-AP; Proteintech, Chicago, IL, USA), rabbit anti-Beclin-1 (cat. no. 3738), and rabbit anti-Atg5 (cat. no. 2630) (both from Cell Signaling Technology, Beverly, MA, USA), rabbit anti-Bcl-2 (cat. no. SC-492; Santa Cruz Biotechnology, Inc.). The blots were washed three times for $5 \mathrm{~min}$ with TBST and then incubated with horseradish peroxidase-labeled secondary antibody for $1 \mathrm{~h}$ at room temperature. Goat anti-mouse IgG (1:2,500; cat. no. SC-2005; Santa Cruz Biotechnology, Inc.) and goat anti-rabbit IgG (1:2500; cat. no. 7074; Cell Signaling Technology) were used as the secondary antibodies. After additional washes, the signal was detected using a West Save Gold western blot detection kit (Youngin Frontier, Seoul, Korea). The protein signals were visualized by exposing the membranes in a luminescent image analyzer (LAS-3000; Fujifilm, Tokyo, Japan). The level of expression of each protein was normalized to that of GAPDH (Sigma-Aldrich). The results were quantified using Multi Gauge, version 3.0 software (Science Laboratory, Tokyo, Japan).

Statistical analysis. The results are presented as mean \pm standard error of the mean (SEM). Statistical comparisons were made with a one-way ANOVA and the Tukey multiple comparison test using GraphPad Prism software, version 5.01 (GraphPad Software Inc., San Diego, CA, USA) to identify significant differences. P-values $<0.05$ were considered statistically significant and P-values $<0.001$ were considered very significant. All experiments were performed at least three times.

\section{Results}

Expression profiling of differentiated myogenic cells derived from T-MSCs. T-MSCs have already been reported to have the characteristics of MSCs $(1,2,21)$. To monitor the molecular events in myogenically differentiated T-MSCs, we 

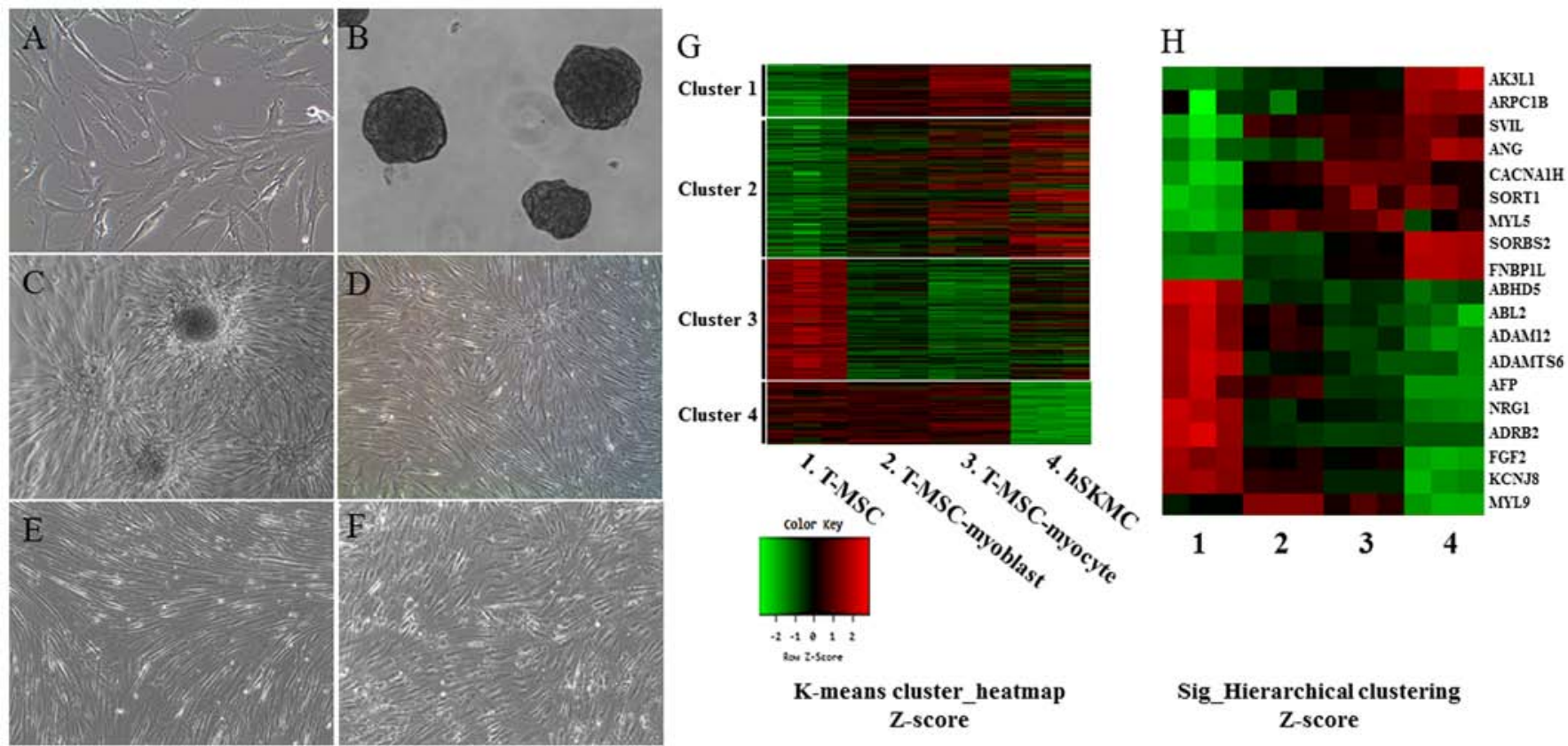

Figure 1. Myogenic differentiation of human T-MSCs and gene expression analysis by microarray. Undifferentiated T-MSCs (A) were induced to form spheres 50-100 mm in diameter (B) in a Petri dish. (C) The T-MSCs expanded beyond the spheres when transferred to a collagen-coated dish in replating medium and formed a rosette-like spread. (D) The plated cells cultured for 4 days in myoblast induction medium. (E) The myoblasts after 14 days in myocyte induction medium showing their altered morphology; they underwent fusion to generate nascent myotubes. (F) hSKMCs cultured for 4 days in serum-free medium containing IGF1. Original magnification, x100. (G) Heatmap of global mRNA expression comparing undifferentiated T-MSCs (sample 1), T-MSC-derived myoblasts (sample 2), T-MSC-derived myocytes (sample 3) and hSKMCs (sample 4). (H) Myogenic gene profile and unsupervised clustering based on markers associated with muscle for undifferentiated T-MSCs, differentiated myogenic cells, and hSKMCs. Lanes 1-4 indicate undifferentiated T-MSCs (sample 1), differentiated myogenic cells (sample 2, T-MSC-myoblast; sample 3, T-MSC-myocyte) and hSKMCs (sample 4). Red indicates upregulated genes and green indicates downregulated genes in G and H. T-MSCs, tonsil-derived mesenchymal stem cells; hSKMCs, human skeletal muscle cells; IGF1, insulin-like growth factor 1.

performed gene expression profiling using microarrays. To obtain RNA samples, the undifferentiated T-MSCs (Fig. 1A) were induced to form spheres in Petri dishes (Fig. 1B) and then the cells expanded beyond the spheres when transferred to a collagen-coated dish in a myoblast induction medium (Fig. 1C), and were cultured for 4 days in myoblast induction medium (Fig. 1D). The T-MSC-derived myoblasts were differentiated for 14 days in myocyte induction medium and their morphology was altered; they fused with one another to generate nascent myotubes (Fig. 1E). As a positive control for myogenic differentiation, hSKMCs (Fig. 1F) that were cultured for 4 days in serum-free medium containing IGF1 were sampled. Global mRNA microarray analysis of T-MSCs was performed at three stages of myogenic differentiation (T-MSC, T-MSC-derived myoblasts and myocytes) and hSKMCs. The raw data (47,323 probes) were tested and 19,765 probes were filtered, normalized, and replicated clones merged and were further taken into account. Subsequently, we found the expression of 3,755 unique genes to be significantly different between the four clusters on the whole $(\mathrm{P}<0.05$; LPE test). The clusters featured four primary expression patterns: an increase in mRNA level during differentiation only (cluster 1); an increase in mRNA level during the differentiation of T-MSCs and hSKMCs (cluster 2); a decrease in mRNA level during the differentiation of T-MSCs and hSKMCs (cluster 3); and a decrease in mRNA level only at the hSKMCs (cluster 4). A heatmap (Fig. 1G) showed that the gene expression was increased (cluster 1) and maintained (cluster 4) compared with T-MSCs during myogenic differentiation, regardless of the gene expression by hSKMCs. Therefore, the genes expressed in cluster 1 and 4 were not associated with the genes expressed by hSKMCs. However, the overall trends of microarray profiles of T-MSC-derived myoblasts and myocytes were similar to those of hSKMCs and quite different from those of undifferentiated T-MSCs (clusters 2 and 3) (Fig. 1G). Furthermore, myogenically differentiated T-MSCs showed a trend of increase or decrease of selected muscle-associated gene expression, similar to hSKMCs (Fig. 1H).

Enrichment of functional categories within muscle metabolism-related genes. The present study classified 3,755 genes into functional categories according to muscle metabolism. Ultimately, 19 known genes related to muscle differentiation were selected, which were upregulated and downregulated in T-MSC-derived myogenic cells and hSKMCs compared with T-MSCs (Tables I and II). These genes were: adenylate kinase 3-like 1 ( $A K 3 L 1)$; actin-related protein $2 / 3$ complex, subunit 1B $(A R P C I B)$; supervillin (SVIL); formin-binding protein 1-like (FNBPIL); angiogenin $(A N G)$; calcium channel, voltage-dependent, T-type, a1H subunit $(C A C N A l H)$; sortilin 1 (SORT1); myosin, light chain 5 (MYL5); sorbin and SH3 domain containing 2 (SORBS2); abhydrolase domain containing 5 (ABHD5); v-abl Abelson murine leukemia viral oncogene homolog 2 (ABL2); ADAM metallopeptidase domain 12 (ADAM12); ADAM metallopeptidase with thrombospondin type 1 motif 6 (ADAMTS6); $\alpha$-fetoprotein $(A F P)$; neuroregulin 1 (NRG1); adrenergic, $\beta 2$, receptor, surface (ADRB2); (fibroblast growth factor 2 ( $F G F 2)$; potas- 
Table I. List of upregulated myogenic genes in the T-MSC-derived myocytes.

\begin{tabular}{|c|c|c|c|c|c|c|}
\hline \multirow[b]{2}{*}{$\begin{array}{l}\text { Functional } \\
\text { category }\end{array}$} & \multirow[b]{2}{*}{ Gene } & \multirow[b]{2}{*}{$\begin{array}{l}\text { Accession no. } \\
\text { symbol }\end{array}$} & \multirow[b]{2}{*}{ Gene name } & \multicolumn{3}{|c|}{ Fold-change (per T-MSC) } \\
\hline & & & & $\begin{array}{l}\text { T-MSC-derived } \\
\text { myoblasts }\end{array}$ & $\begin{array}{l}\text { T-MSC-derived } \\
\text { myocytes }\end{array}$ & SKMCs \\
\hline \multirow{3}{*}{$\begin{array}{l}\text { Muscle tissue } \\
\text { development }\end{array}$} & $A K 3 L 1$ & NM_203464.1 & Adenylate kinase 3 -like 1 & 1.39 & 1.63 & 3.89 \\
\hline & $A R P C 1 B$ & NM_005720.2 & $\begin{array}{l}\text { Actin related protein } 2 / 3 \\
\text { complex, subunit } 1 \mathrm{~B}\end{array}$ & 1.18 & 1.67 & 2.71 \\
\hline & SVIL & NM_003174.3 & Supervillin & 2.43 & 2.43 & 2.77 \\
\hline Autophagy & $F N B P 1 L$ & NM_001024948.1 & $\begin{array}{l}\text { Formin binding } \\
\text { protein 1-like }\end{array}$ & 1.95 & 3.49 & 12.97 \\
\hline $\begin{array}{l}\text { Negative regulation } \\
\text { of smooth muscle } \\
\text { cell proliferation }\end{array}$ & $A N G$ & NM_003174.3 & $\begin{array}{l}\text { Angiogenin, ribonuclease, } \\
\text { RNase A family, } 5\end{array}$ & 1.21 & 1.88 & 2.45 \\
\hline \multirow[t]{2}{*}{$\begin{array}{l}\text { Muscle cell } \\
\text { differentiation }\end{array}$} & CACNAIH & NM_021098.2 & $\begin{array}{l}\text { Calcium channel, } \\
\text { voltage-dependent, } \\
\text { T type, } \alpha 1 \mathrm{H} \text { subunit }\end{array}$ & 1.93 & 2.45 & 2.02 \\
\hline & SORT1 & NM_002959.4 & Sortilin 1 & 1.76 & 2.35 & 2.33 \\
\hline \multirow[t]{2}{*}{ Actin cytoskeleton } & MYL5 & NM_002477.1 & $\begin{array}{l}\text { Myosin, light } \\
\text { chain } 5\end{array}$ & 1.95 & 2.02 & 1.56 \\
\hline & SORBS2 & NM_003603.4 & $\begin{array}{l}\text { Sorbin and SH3 } \\
\text { domain containing } 2\end{array}$ & 1.57 & 4.04 & 30.82 \\
\hline
\end{tabular}

Table II. List of downregulated myogenic genes in the T-MSC-derived myocytes.

Fold-change (per T-MSC)

\begin{tabular}{|c|c|c|c|c|c|c|}
\hline $\begin{array}{l}\text { Functional } \\
\text { category }\end{array}$ & Gene & $\begin{array}{l}\text { Accession no. } \\
\text { symbol }\end{array}$ & Gene name & $\begin{array}{l}\text { T-MSC-derived } \\
\text { myoblasts }\end{array}$ & $\begin{array}{l}\text { T-MSC-derived } \\
\text { myocytes }\end{array}$ & SKMCs \\
\hline \multirow[t]{6}{*}{$\begin{array}{l}\text { Muscle tissue } \\
\text { development }\end{array}$} & ABHD5 & NM_016006.3 & $\begin{array}{l}\text { Abhydrolase domain } \\
\text { containing } 5\end{array}$ & -2.09 & -2.14 & -2.37 \\
\hline & ABHD5 & NM_007314.2 & $\begin{array}{l}\text { v-abl Abelson murine } \\
\text { leukemia viral } \\
\text { oncogene homolog } 2\end{array}$ & -1.38 & -1.69 & -2.06 \\
\hline & $A D A M 12$ & NM_021641.2 & $\begin{array}{l}\text { ADAM metallopeptidase } 12 \\
\text { domain }\end{array}$ & -1.40 & -1.76 & -2.16 \\
\hline & ADAMTS6 & NM_197941.2 & $\begin{array}{l}\text { ADAM metallopeptidase } \\
\text { with thrombospondin } \\
\text { type } 1 \text { motif } 6\end{array}$ & -1.84 & -2.07 & -2.38 \\
\hline & $A F P$ & NM_001134.1 & $\alpha$-fetoprotein & -1.41 & -2.03 & -2.87 \\
\hline & $N R G 1$ & NM_013962.2 & Neuregulin 1 & -2.42 & -2.41 & -3.70 \\
\hline $\begin{array}{l}\text { Regulation of } \\
\text { muscle organ }\end{array}$ & $A D R B 2$ & NM_000024.3 & $\begin{array}{l}\text { Adrenergic, } \beta-2- \\
\text { receptor, surface }\end{array}$ & -4.02 & -4.50 & -5.21 \\
\hline development & $F G F 2$ & NM_002006.3 & $\begin{array}{l}\text { Fibroblast growth factor } 2 \\
\text { (basic) }\end{array}$ & -1.95 & -2.04 & -5.30 \\
\hline Myofibril & KCNJ8 & NM_004982.2 & $\begin{array}{l}\text { Potassium inwardly- } \\
\text { rectifying channel, } \\
\text { subfamily } \mathbf{J}, \text { member } 8\end{array}$ & -1.67 & -2.42 & -4.31 \\
\hline Actin cytoskeleton & $M Y L 9$ & NM_006097.3 & $\begin{array}{l}\text { Myosin, light chain } 9 \text {, } \\
\text { regulatory }\end{array}$ & 2.00 & 1.34 & -2.15 \\
\hline
\end{tabular}

T-MSC, tonsil-derived mesenchymal stem cell; SKMC, skeletal muscle cells. 

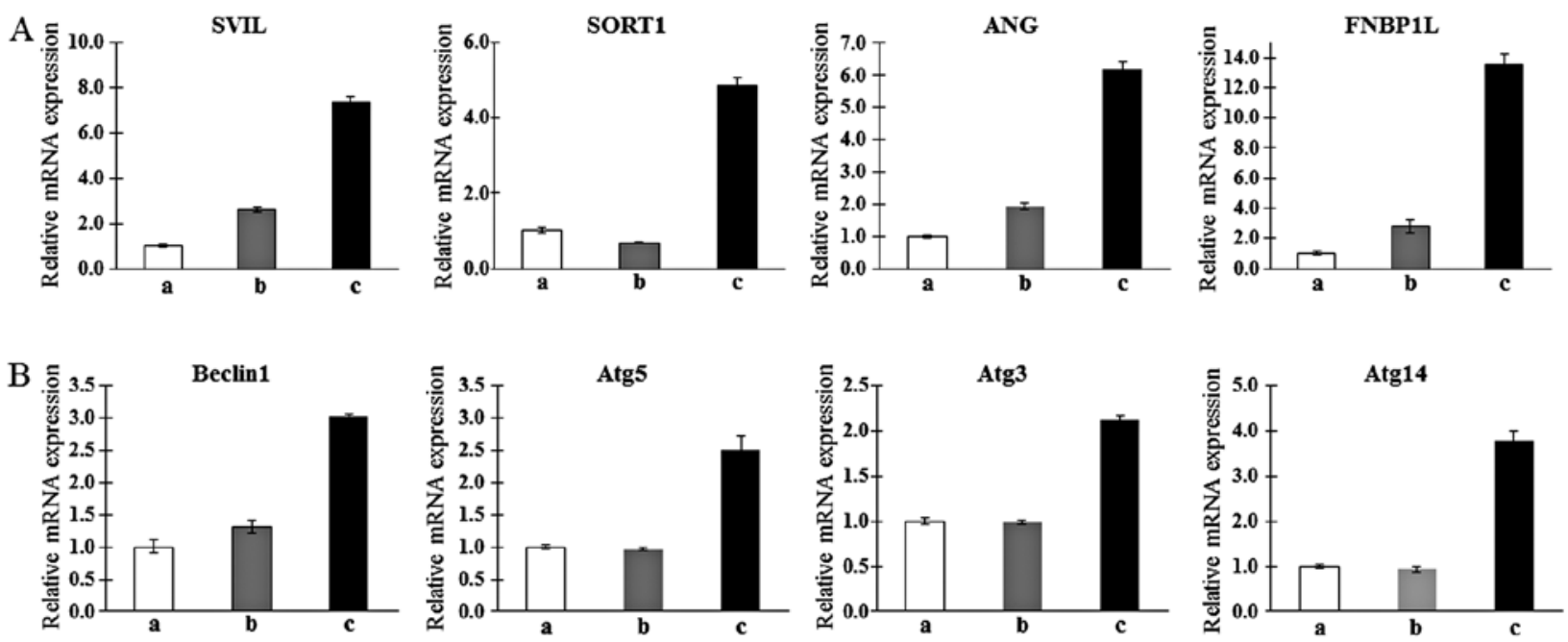

Figure 2. Determination of the mRNA expression levels of upregulated muscle metabolism (A) and autophagy-related markers (B) as myogenic markers in human T-MSCs during the process of myogenic induction (a, T-MSCs; b, T-MSC-derived myoblasts; c, T-MSC-derived myocytes). GAPDH served as a loading control. Data are expressed as mean \pm standard error of the mean (SEM). Triplicate independent mRNA samples were used in an RT-PCR experiment. T-MSCs, tonsil-derived mesenchymal stem cells; SVIL, supervillin; SORT1, sortilin 1; ANG, angiogenin; FNBPIL, formin binding protein 1-like; Atg5, autophagy-related gene 5; Atg3, autophagy-related gene 3; Atg14, autophagy-related gene 14; GAPDH, glyceraldehyde 3-phosphate dehydrogenase.
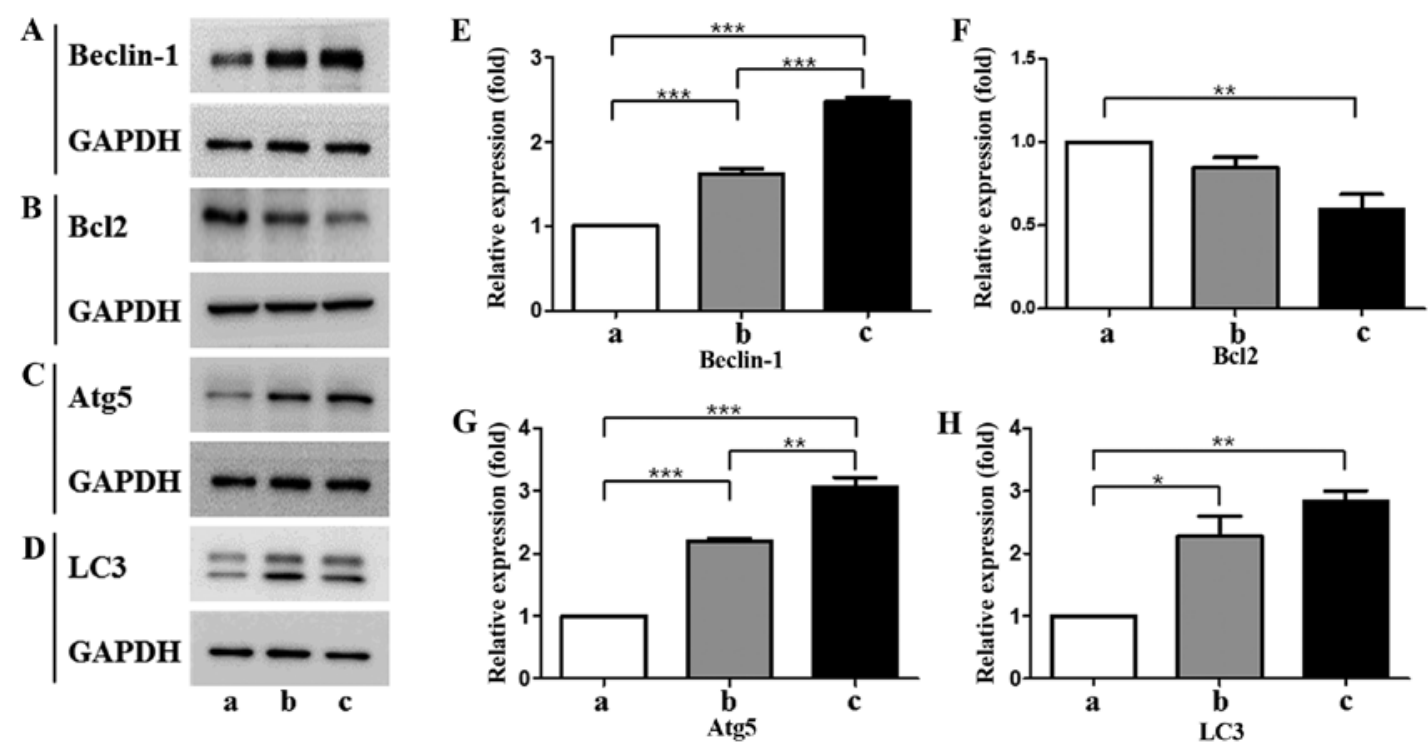

Figure 3. (A-D) Protein expression levels and (E-H) quantification of autophagic markers in T-MSCs during the process of myogenic induction (a, T-MSCs; b, T-MSC-derived myoblasts; c, T-MSC-derived myocytes). The levels of GAPDH were measured as a loading control (A-D). All signals were analyzed by densitometric scanning [(E-H) LAS-3000; Fujifilm, Tokyo, Japan)]. Data are expressed as means \pm SEM of experiments performed in triplicate. ${ }^{*}<0.05$, ${ }^{* *} \mathrm{P}<0.01$ and ${ }^{* * * *} \mathrm{P}<0.001$. T-MSCs, human tonsil-derived mesenchymal stem cells; Bcl-2, B cell lymphoma 2; Atg5, autophagy-related gene 5; LC3, microtubuleassociated protein 1A/1B-light chain 3; GAPDH, glyceraldehyde 3-phosphate dehydrogenase.

sium inwardly-rectifying channel, subfamily J, member 8 (KCNJ8); myosin, light chain 9, regulatory (MYL9). These are related to muscle cell differentiation, muscle tissue and organ development, autophagy, regulation of muscle organ development, myofibrils, actin cytoskeleton, and negative regulation of smooth muscle cell proliferation. Moreover, the expression level (fold-change) in the T-MSCs of those genes was found to be different in the three cell types $(\mathrm{P}<0.05$; LPE test). Thus, we were able to verify that the properties of the T-MSCderived myocytes were more similar to those of hSKMCs than T-MSC-derived myoblasts. In particular, formin binding protein 1-like (FNBP1L), which has an essential role in antibacterial autophagy, was selected among the upregulated genes, since autophagy is related to skeletal muscle metabolism and myogenesis. Furthermore, FNBP1L has not been reported in autophagy signaling in stem cells. As a result, we demonstrated the effect of autophagy in the sequential differentiating of T-MSCs toward skeletal myocytes in vitro.

Confirmation of gene expression profiles of T-MSC-derived myogenic cells. To confirm the microarray findings and their relationship with autophagy, qPCR was analyzed. Consistent with the results of the microarray analysis, candidate genes associated with myogenic differentiation such as SVIL (22), SORTI (23), ANG (24) and FNBPIL (25), which are muscle metabolism-related genes, were upregulated in 

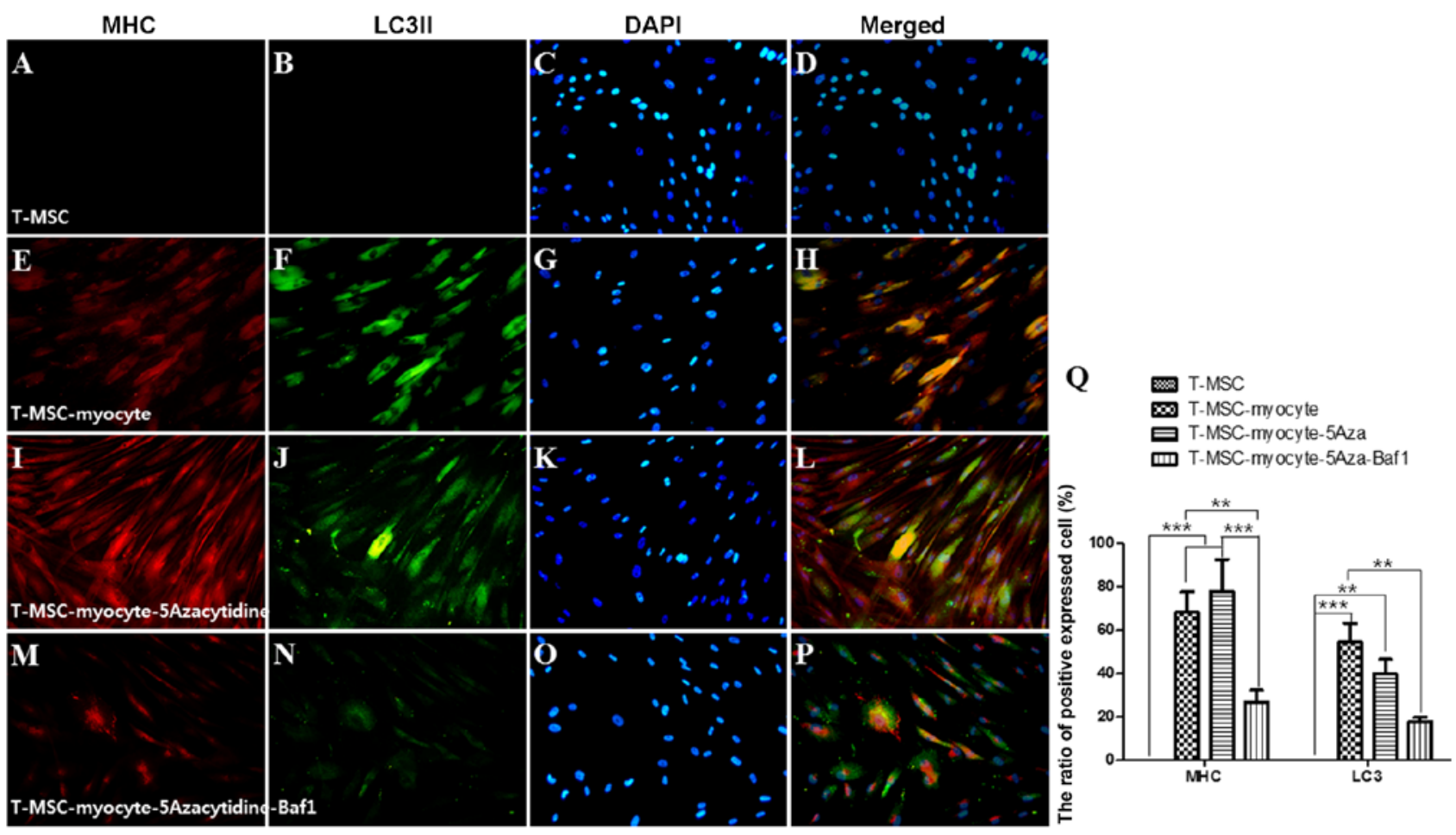

Figure 4. Effect of 5Aza and Baf1 on the skeletal myogenic differentiation of T-MSCs. The expression of MHC (red) and LC3II (green) in the T-MSCs (A-D) during the process of myogenic differentiation [T-MSC-derived myocytes (E-H)]. The role of autophagy was evaluated with an inducer, 5Aza [T-MSC-derived myocytes-5-azacytidine (I-L)], and an inhibitor, Baf1 [T-MSC-derived myocytes-5-azacytidine-Baf1 (M-P)], during skeletal myogenic differentiation. The cells were counterstained with DAPI (blue). The samples were analyzed under a fluorescence microscope using appropriate filters. Original magnification, x200. (Q) The ratio of cells immunoreactive for anti-MHC and anti-LC3. Data are expressed as means \pm SEM. For each condition, four slides were used for quantification. Graphs represent the average of multiple tests from three independent experiments. 5Aza, 5-azacytidine; Baf1, bafilomycin A1; MHC, myosin heavy chain; LC3II, microtubule-associated protein 1A/1B-light chain 3; T-MSCs, tonsil-derived mesenchymal stem cells.

the T-MSC-derived myoblasts and myocytes, but not in the undifferentiated T-MSCs with the exception of SORT1. The expression of SORT1 was only upregulated in T-MSC-derived myocytes (Fig. 2A). In particular, FNBPIL was expected to be the instrument for tracking the relationship between autophagy and differentiation into myocytes. We therefore confirmed the higher expression of autophagy-related genes such as beclin-1, Atg5, Atg3 and Atg14 in the T-MSC-derived myocytes compared with expression in the T-MSCs or T-MSC-derived myoblasts (Fig. 2B). Taken together, T-MSC-derived myogenic cells appeared to have characteristics similar to differentiated hSKMCs and affect the mechanism of autophagy.

Expression of autophagy-related genes under states that induce myogenic differentiation. As determine by western blotting, expression of Beclin-1 and Atg5 was increased gradually from T-MSC-derived myoblasts to myocytes (Fig. 3A, C, E and G). However, the expression of Bcl-2, which binds with beclin-1 resulting in the inhibition of autophagic initiation (26), was decreased at all stages of differentiation (Fig. 3B and F). The expression of LC3II, which is a well-characterized protein specifically localized to autophagic structures throughout the process from phagophores to lysosomal degradation (27), was increased gradually from T-MSC-derived myoblasts to myocytes, but was not significantly different between the T-MSC-derived myoblast and myocyte stages (Fig. 3D and H). These results indicate that myogenic differentiation of T-MSCs is associated with the autophagic pathway.
Detection of the role of autophagy in T-MSC-derived myocytes by immunostaining. We used immunocytochemistry to investigate whether autophagy participates in myogenic differentiation of T-MSCs. To measure their innate ability to differentiate into myocytes, T-MSC-derived myoblasts were plated for myogenic differentiation to the skeletal myocytes onto coverslips and cultured sequentially in myogenic differentiation medium, and treated with an autophagy inducer (5-azacytidine) and inhibitor (Baf1). These cells were then fixed and co-labeled with antibodies against the markers of autophagy (LC3) and skeletal myogenic cells (MHC, myogenin, desmin), respectively. Following differentiation into myocytes, $60-70 \%$ of the cells expressed MHC (Fig. 4E), myogenin (Fig. 5E), and desmin (Fig. 6E), and $45-55 \%$ of the cells co-expressed autophagic marker LC3II (Figs. 4F, 5F and 6F). 5-Azacytidine was added to T-MSC-derived myoblasts to activate autophagy for powerful myogenic differentiation. When treated with 5-azacytidine, the expression of myogenic markers was increased (67-77\%) (Figs. 4I, 5I and 6I), while LC3 was not significantly increased $(40-55 \%)$ (Figs. 4J, 5J and $6 \mathrm{~J})$. By contrast, the expression of myogenic markers was decreased after treatment with Baf1 during the differentiation of T-MSC-derived myocytes (13-32\%) (Figs. 4M, 5M and $6 \mathrm{M}$ ) and LC3II was significantly more decreased than in T-MSC-derived myocytes and T-MSC-derived myocytes treated with 5-azacytidine (16-23\%) (Figs. 4N, 5N and 6N). These results showed that autophagy has an effect on the mechanism of skeletal myogenic differentiation in T-MSCs. 


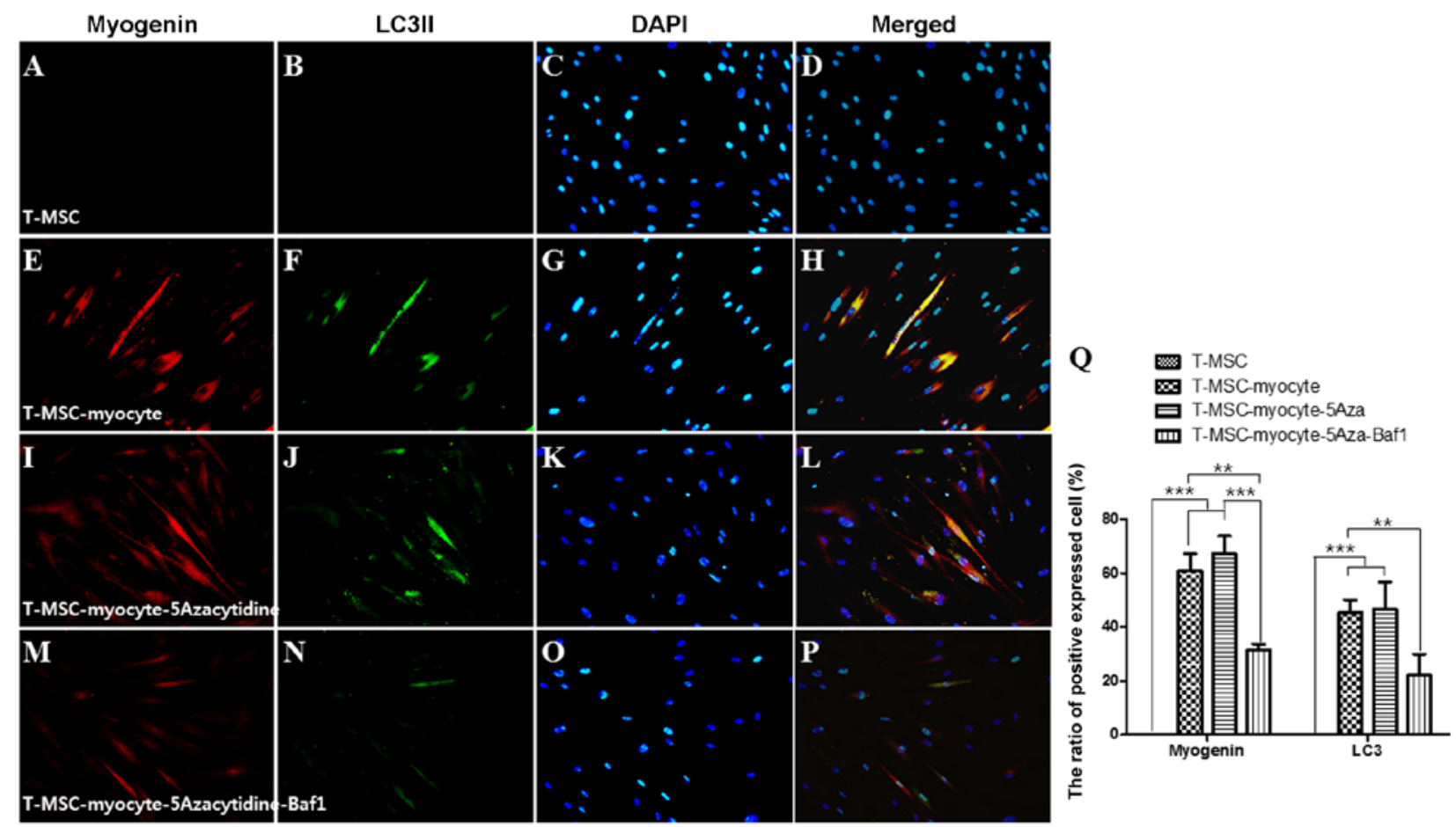

Figure 5. Effect of 5Aza and Baf1 on the skeletal myogenic differentiation of T-MSCs. The expression of myogenin and LC3II (green) in the T-MSCs (A-D) during the process of myogenic differentiation [T-MSC-derived myocytes (E-H)]. The role of autophagy was evaluated with an inducer, 5Aza [T-MSC-derived myocytes-5-azacytidine (I-L)], and an inhibitor, Baf1 [T-MSC-derived myocytes-5-azacytidine-Baf1 (M-P)] during skeletal myogenic differentiation. The cells were counterstained with DAPI (blue). The samples were analyzed under a fluorescence microscope using appropriate filters. Original magnification, x200. (Q) The ratio of cells immunoreactive for anti-myogenin and anti-LC3. Data are expressed as means \pm SEM. For each condition, four slides were used for quantification. Graphs represent the average of multiple tests from three independent experiments. 5Aza, 5-azacytidine; Baf1, bafilomycin A1; LC3II, microtubule-associated protein 1A/1B-light chain 3; T-MSCs, tonsil-derived mesenchymal stem cells.

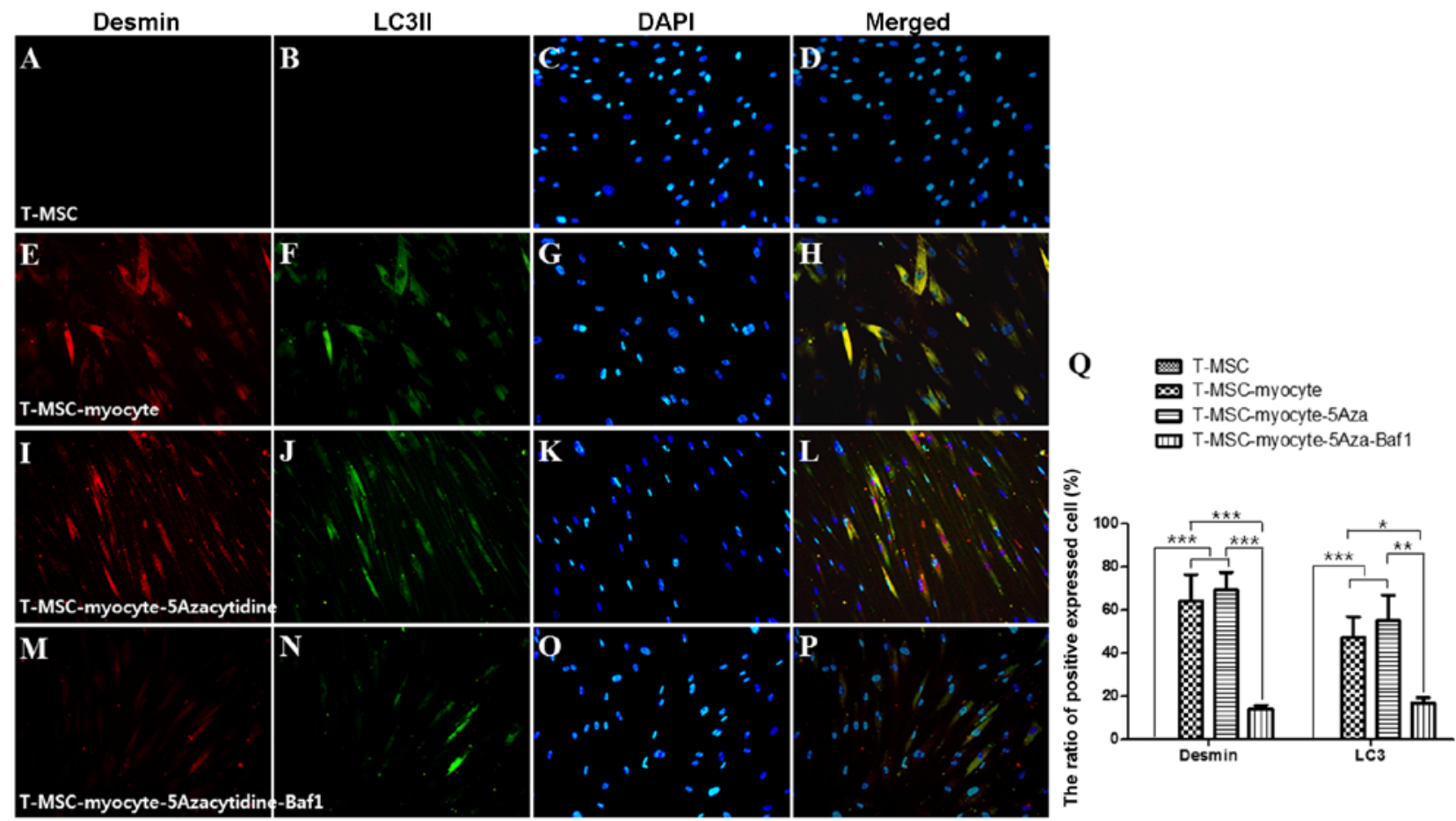

Figure 6. Effect of 5Aza and Baf1 on the skeletal myogenic differentiation of human T-MSCs. The expression of desmin and LC3II (green) in the T-MSCs (A-D) during the process of myogenic differentiation [T-MSC-derived myocytes; (E-H)]. The role of autophagy was evaluated with an inducer, 5Aza [T-MSC-derived myocytes-5-azacytidine (I-L)], and an inhibitor, Baf1 [T-MSC-derived myocytes-5-azacytidine-Baf1 (M-P)], during skeletal myogenic differentiation. The cells were counterstained with DAPI (blue). The samples were analyzed under a fluorescence microscope using appropriate filters. Original magnification, $\mathrm{x} 200$. (Q) The ratio of cells immunoreactive for anti-desmin and anti-LC3. Data are expressed as means \pm SEM. For each condition, four slides were used for quantification. Graphs represent the average of multiple tests from three independent experiments. 5Aza, 5-azacytidine; Baf1, bafilomycin A1; LC3II, microtubule-associated protein 1A/1B-light chain 3; Baf1, bafilomycin A1; T-MSCs, tonsil-derived mesenchymal stem cells. 


\section{Discussion}

Autophagy plays an essential role in the differentiation of human bone marrow MSCs into osteocytes and adipocytes (18). Mitophagy is required for mitochondrial biogenesis and myogenic differentiation of $\mathrm{C} 2 \mathrm{C} 12$ myoblasts (19). The microarray analysis followed by immunocytochemistry used in the present study demonstrated that autophagy is related to the differentiation of T-MSCs into myocytes.

Microarray analysis revealed the potential for the differentiation of T-MSCs into skeletal myocytes. All clusters expressed cell proliferation and motion-related genes in common (Fig. 1G). In cluster 2, the muscle cell differentiation and tissue development-related genes were highly expressed, and the gene expression was gradually increased from the differentiated T-MSCs to SKMCs. The genes of cluster 3 showed a tendency for decreased expression and were associated with the cell cycle or components within the cell and smooth or cardiac muscle markers. Related to cell cycle progression and metabolism, these genes are downregulated upon the initiation of myogenic differentiation (28). The genes related to mesenchymal or MSC development or gastrulation were particularly expressed in cluster 4, which showed a pattern of high gene expression except for SKMCs. It can be assumed that the genes of cluster 4 are associated with the characteristics of stem cells. In addition, all the clusters, except cluster 4, expressed mesoderm or angiogenesis-related genes. In cluster 1 , gene expression was increased in the differentiating T-MSCs (T-MSC-derived myoblasts and myocytes) and only the TGF- $\beta$ or collagen gene was expressed. TGF- $\beta$ and collagen were used to differentiate myoblasts in the present study. TGF- $\beta$ and collagen have a role in the differentiation of the stem cells to myoblasts $(1,29,30)$.

We selected 9 upregulated genes and 10 downregulated genes in the T-MSC-derived myogenic cells and hSKMCs compared with T-MSCs as related to muscle differentiation (Tables I and II). Among the 19 genes selected, several genes (SVIL, SORT1, ANG and FNBPIL) appeared as important factors in the mechanism of skeletal myogenic differentiation, as the RT-PCR analysis data were consistent with the microarray data. The expression of all of these genes was significantly increased in the differential stage in the T-MSC-derived myocytes. Supervillin (SVIL) is implicated in the direct or indirect control of cell adhesion (22) and localizes within nuclei and with dystrophin at costameres, regions of F-actin membrane attachment in skeletal muscle (31). Archvillin, a muscle-specific isoform of SVIL, is among the first costameric proteins to assemble during myogenesis and it contributes to myogenic membrane structure and differentiation. Sortilin 1 (SORT1) was originally purified from human brain extracts and has been identified as a major component of GLUT4-containing vesicles from rat adipocytes (23). SORT1 also has functional roles in the development of the insulin-responsive glucose uptake system in muscle cells (32). Angiogenin (ANG) is involved in blood vessel formation. However, ANG was originally shown to bind to a $42-\mathrm{kDa}$ binding protein and a smooth muscle-type $\alpha$-actin before the identification of endothelial receptors. ANG has been demonstrated in human arterial smooth muscle cell prolifera- tion, through the binding of $\alpha$-actinin-2, which is traditionally designated as an SKM isoform due to its presence in both skeletal and cardiac muscles $(24,33)$. FNBP1L is essential for antibacterial autophagy and bridges the autophagic membrane extension machinery and its bacterial cargo. An autophagosome is built around bacterial cargo by a coordinated series of autophagy protein complexes; therefore, the HR1 domain of FNBP1L is biochemically essential for its interaction with human Atg3 protein (25). The pathway of these genes (FNBPIL and Atg3) and Atg5 causes the Atg12-Atg5 conjugate to interact with Atg3 (34), which is consistent with our results in which the gene expression was increased in T-MSC-derived myocytes (Fig. 2).

Nutrients are the main physiological regulators and initiators of autophagy; therefore, the lack of nutrients is an important inducer of SKM autophagy (35). In the present study, we used a method that minimizes serum in the culture medium to differentiate stem cells into skeletal myocytes. Autophagy is induced by such a fasting method and in turn promotes myogenic differentiation. 5-Azacytidine is already used as a factor for the differentiation of muscle stem cells into muscle cells, and autophagy has relevance to myogenic differentiation $(36,37)$.

Three main pathways (macrophagy, microphagy and chaperone-mediated autophagy) are involved in autophagy and these are mediated by autophagy-related genes and their enzymes (38). Levels of proteins including Beclin-1, Bcl-2, LC3 and Atg5 were increased and decreased in the present study, and they are the key factors in the autophagy signal pathway related to macrophagy. Depending on the myogenic differentiation of T-MSCs, the expression of Beclin-1,LC3 and Atg5 was increased, while Bcl-2 was decreased. LC3 was coexpressed with myogenic markers (MHC, myogenin and desmin) in the T-MSC-derived myocytes, which was confirmed by the treatment of T-MSC-derived myocytes with an inducer and an inhibitor. The role of autophagy as a metabolic regulator in muscle is well known. In particular, macrophagy represents the physiological process that skeletal muscle uses to transport cytoplasm, organelles, and proteins to lysosomes for degradation (39). Various SKM diseases that cause atrophy and dystrophy have shed light on a significant common feature, namely the build-up of autophagosomes within myofibers (40). Comparing our study with these previous reports, autophagy appears to influence skeletal myogenic differentiation of T-MSCs.

In the present study, we classified genes into functional categories according to muscle metabolism, and specific genes related to muscle differentiation were selected in the T-MSC-derived myogenic cells and hSKMCs. Functional studies demonstrated that autophagy plays a role during the differentiation of T-MSCs into hSKMCs. Furthermore, as a result of treatment of T-MSC-derived myocytes with an autophagy inducer and inhibitor, we were able to reconfirm that autophagy has an effect on the mechanism of myogenic differentiation of T-MSCs. Our study suggests that autophagy plays an important role during the differentiation of T-MSCs into skeletal myogenic cells and knowledge of this mechanism is valuable for treating SKM injuries and the damage caused by other degenerative disorders, including congenital defects, trauma or tumor removal. 


\section{Acknowledgements}

The present study was supported by a grant (HI12C0135) from the Korean Health Technology R\&D Project, Ministry of Health and Welfare, Republic of Korea and intramural research promotion grants from Ewha Womans University School of Medicine.

\section{References}

1. Ryu KH, Cho KA, Park HS, Kim JY, Woo SY, Jo I, Choi YH, Park YM, Jung SC, Chung SM, et al: Tonsil-derived mesenchymal stromal cells: Evaluation of biologic, immunologic and genetic factors for successful banking. Cytotherapy 14: 1193-1202, 2012.

2. Park S, Choi Y, Jung N, Yu Y, Ryu KH, Kim HS, Jo I, Choi BO and Jung SC: Myogenic differentiation potential of human tonsil-derived mesenchymal stem cells and their potential for use to promote skeletal muscle regeneration. Int J Mol Med 37: 1209-1220, 2016.

3. Dezawa M, Ishikawa H, Itokazu Y, Yoshihara T, Hoshino M, Takeda S, Ide C and Nabeshima Y: Bone marrow stromal cells generate muscle cells and repair muscle degeneration. Science 309: 314-317, 2005.

4. Di Rocco G, Iachininoto MG, Tritarelli A, Straino S, Zacheo A, Germani A, Crea F and Capogrossi MC: Myogenic potential of adipose-tissue-derived cells. J Cell Sci 119: 2945-2952, 2006.

5. Kim JA, Shon YH, Lim JO, Yoo JJ, Shin HI and Park EK: MYOD mediates skeletal myogenic differentiation of human amniotic fluid stem cells and regeneration of muscle injury. Stem Cell Res Ther 4: 147, 2013.

6. Nunes VA, Cavaçana N, Canovas M, Strauss BE and Zatz M Stem cells from umbilical cord blood differentiate into myotubes and express dystrophin in vitro only after exposure to in vivo muscle environment. Biol Cell 99: 185-196, 2007.

7. Park S, Kim E, Koh SE, Maeng S, Lee WD, Lim J, Shim I and Lee YJ: Dopaminergic differentiation of neural progenitors derived from placental mesenchymal stem cells in the brains of Parkinson's disease model rats and alleviation of asymmetric rotational behavior. Brain Res 1466: 158-166, 2012.

8. Kerkis I, Kerkis A, Dozortsev D, Stukart-Parsons GC, Gomes Massironi SM, Pereira LV, Caplan AI and Cerruti HF: Isolation and characterization of a population of immature dental pulp stem cells expressing OCT-4 and other embryonic stem cell markers. Cells Tissues Organs 184: 105-116, 2006.

9. Husmann I, Soulet L, Gautron J, Martelly I and Barritault D: Growth factors in skeletal muscle regeneration. Cytokine Growth Factor Rev 7: 249-258, 1996.

10. Wang $\mathrm{K}$, Wang $\mathrm{C}$, Xiao F, Wang $\mathrm{H}$ and Wu Z: JAK2/STAT2/ STAT3 are required for myogenic differentiation. J Biol Chem 283 : 34029-34036, 2008

11. Conboy IM and Rando TA: The regulation of Notch signaling controls satellite cell activation and cell fate determination in postnatal myogenesis. Dev Cell 3: 397-409, 2002.

12. Fortini P, Ferretti C, Iorio E, Cagnin M, Garribba L, Pietraforte D, Falchi M, Pascucci B, Baccarini S, Morani F, et al: The fine tuning of metabolism, autophagy and differentiation during in vitro myogenesis. Cell Death Dis 7: e2168, 2016.

13. Maley MA, Fan Y, Beilharz MW and Grounds MD: Intrinsic differences in MyoD and myogenin expression between primary cultures of SJL/J and BALB/C skeletal muscle. Exp Cell Res 211: 99-107, 1994.

14. Miller KJ, Thaloor D, Matteson S and Pavlath GK: Hepatocyte growth factor affects satellite cell activation and differentiation in regenerating skeletal muscle. Am J Physiol Cell Physiol 278 C174-C181, 2000.

15. Heszele MF and Price SR: Insulin-like growth factor I: The yin and yang of muscle atrophy. Endocrinology 145: 4803-4805, 2004.

16. Nakanishi K, Dohmae N and Morishima N: Endoplasmic reticulum stress increases myofiber formation in vitro. FASEB J 21: 2994-3003, 2007

17. Levine B and Klionsky DJ: Development by self-digestion: Molecular mechanisms and biological functions of autophagy. Dev Cell 6: 463-477, 2004

18. Nuschke A, Rodrigues M, Stolz DB, Chu CT, Griffith L and Wells A: Human mesenchymal stem cells/multipotent stromal cells consume accumulated autophagosomes early in differentiation. Stem Cell Res Ther 5: 140, 2014.
19. Sin J, Andres AM, Taylor DJ, Weston T, Hiraumi Y, Stotland A, Kim BJ, Huang C, Doran KS and Gottlieb RA: Mitophagy is required for mitochondrial biogenesis and myogenic differentiation of C2C12 myoblasts. Autophagy 12: 369-380, 2016.

20. Livak KJ and Schmittgen TD: Analysis of relative gene expression data using real-time quantitative PCR and the 2(-Delta Delta C(T)) method. Methods 25: 402-408, 2001.

21. Yu Y, Park YS, Kim HS, Kim HY, Jin YM, Jung SC, Ryu KH and Jo I: Characterization of long-term in vitro culture-related alterations of human tonsil-derived mesenchymal stem cells: Role for CCN1 in replicative senescence-associated increase in osteogenic differentiation. J Anat 225: 510-518, 2014.

22. Pestonjamasp KN, Pope RK, Wulfkuhle JD and Luna EJ: Supervillin (p205): A novel membrane-associated, F-actin-binding protein in the villin/gelsolin superfamily. J Cell Biol 139: $1255-1269,1997$.

23. Morris NJ, Ross SA, Lane WS, Moestrup SK, Petersen CM, Keller SR and Lienhard GE: Sortilin is the major 110-kDa protein in GLUT4 vesicles from adipocytes. J Biol Chem 273: 3582-3587, 1998

24. Hu H, Gao X, Sun Y, Zhou J, Yang M and Xu Z: Alpha-actinin-2, a cytoskeletal protein, binds to angiogenin. Biochem Biophys Res Commun 329: 661-667, 2005.

25. Huett A, Ng A, Cao Z, Kuballa P, Komatsu M, Daly MJ, Podolsky DK and Xavier RJ: A novel hybrid yeast-human network analysis reveals an essential role for FNBP1L in antibacterial autophagy. J Immunol 182: 4917-4930, 2009.

26. Marquez RT and Xu L: Bcl-2:Beclin 1 complex: Multiple, mechanisms regulating autophagy/apoptosis toggle switch. Am J Cancer Res 2: 214-221, 2012.

27. Nakatogawa H, Suzuki K, Kamada Y and Ohsumi Y: Dynamics and diversity in autophagy mechanisms: Lessons from yeast. Nat Rev Mol Cell Biol 10: 458-467, 2009.

28. Moran JL, Li Y, Hill AA, Mounts WM and Miller CP: Gene expression changes during mouse skeletal myoblast differentiation revealed by transcriptional profiling. Physiol Genomics 10: 103-111, 2002.

29. Osses $\mathbf{N}$ and Brandan E: ECM is required for skeletal muscle differentiation independently of muscle regulatory factor expression. Am J Physiol Cell Physiol 282: C383-C394, 2002.

30. Hinz B, Phan SH, Thannickal VJ, Galli A, Bochaton-Piallat ML and Gabbiani G: The myofibroblast: One function, multiple origins. Am J Pathol 170: 1807-1816, 2007.

31. Oh SW, Pope RK, Smith KP, Crowley JL, Nebl T, Lawrence JB and Luna EJ: Archvillin, a muscle-specific isoform of supervillin, is an early expressed component of the costameric membrane skeleton. J Cell Sci 116: 2261-2275, 2003.

32. Ariga M, Nedachi T, Katagiri H and Kanzaki M: Functional role of sortilin in myogenesis and development of insulin-responsive glucose transport system in $\mathrm{C} 2 \mathrm{C} 12$ myocytes. J Biol Chem 283: 10208-10220, 2008

33. Kostin S, Hein S, Arnon E, Scholz D and Schaper J: The cytoskeleton and related proteins in the human failing heart. Heart Fail Rev 5: 271-280, 2000.

34. Sakoh-Nakatogawa M, Matoba K, Asai E, Kirisako H, Ishii J, Noda NN, Inagaki F, Nakatogawa $\mathrm{H}$ and Ohsumi Y: Atg12-Atg5 conjugate enhances E2 activity of Atg3 by rearranging its catalytic site. Nat Struct Mol Biol 20: 433-439, 2013.

35. Neel BA, Lin Y and Pessin JE: Skeletal muscle autophagy: A new metabolic regulator. Trends Endocrinol Metab 24: 635-643, 2013.

36. Wakitani S, Saito T and Caplan AI: Myogenic cells derived from rat bone marrow mesenchymal stem cells exposed to 5-azacytidine. Muscle Nerve 18: 1417-1426, 1995.

37. Zheng JK, Wang Y, Karandikar A, Wang Q, Gai H, Liu AL, Peng C and Sheng HZ: Skeletal myogenesis by human embryonic stem cells. Cell Res 16: 713-722, 2006.

38. Mizushima N, Ohsumi Y and Yoshimori T: Autophagosome formation in mammalian cells. Cell Struct Funct 27: 421-429, 2002.

39. Jackson MJ: Reactive oxygen species and redox-regulation of skeletal muscle adaptations to exercise. Philos Trans R Soc Lond B Biol Sci 360: 2285-2291, 2005.

40. Malicdan MC, Noguchi S, Nonaka I, Saftig P and Nishino I: Lysosomal myopathies: An excessive build-up in autophagosomes is too much to handle. Neuromuscul Disord 18: 521-529, 2008. 